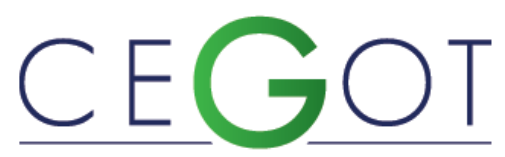

Centro de Estudos de Geografia e Ordenamento do Território

\author{
DUARTE, PAULINO \\ Estudante de Mestrado em Geografia Física e Ordenamento do \\ Território \\ 3004-530, Coimbra, Portugal \\ paulinoerd70@gmail.com
}

Figueiredo, Albano

Universidade Coimbra, Departamento de Geografia e Turismo / Centro

de Estudos em Geografia e Ordenamento do Território

3004-530 Coimbra, Portugal

geofiguc@gmail.com

NUNES, AdÉLIA

Universidade Coimbra, Departamento de Geografia e Turismo / Centro

de Estudos em Geografia e Ordenamento do Território

3004-530 Coimbra, Portugal

adelia.nunes@fl.uc.pt

\title{
Avaliação da influência do tempo de combustão na determinação do carbono orgânico do solo em dois tipos de floresta perenifólia
}

Assessing the influence of combustion time on the determination of soil organic carbon in two types of evergreen forest

Referência: Duarte, Paulino; Figueiredo, Albano; Nunes, Adélia (2020). Avaliação da influência do tempo de combustão na determinação do carbono orgânico do solo em dois tipos de floresta perenifólia. Revista de Geografia e Ordenamento do Território (GOT), n.o 20 (Dezembro). Centro de Estudos de Geografia e Ordenamento do Território, p. 280-293, dx.doi.org/10.17127/got/2020.20.012

\section{RESUMO}

O problema das mudanças climáticas associadas ao aquecimento global promoveu a necessidade de identificação e implementação de medidas de mitigação e adaptação, com o objetivo de reduzir os impactes potenciais. No âmbito destas medidas, a redução de emissões de gases com efeito de estufa tem sido uma das medidas propostas mais recorrentes. No caso do dióxido de carbono, além da redução das emissões, têm ainda sido propostas medidas que visam o aumento da capacidade do seu sequestro no solo. Neste 
contexto, diferentes trabalhos têm avaliado a concentração de carbono orgânico em diferentes condições edáficas e uso do solo, tendo por referência diferentes aproximações metodológicas. Nos estudos em que foi utilizado o método da combustão, diferentes parâmetros foram usados ao nível da duração e temperatura da combustão, o que não contribui para a possibilidade de uma comparação clara entre resultados. Este trabalho procura avaliar se o tempo de combustão interfere de forma significativa nos resultados obtidos para diferentes tipos de solo considerando dois tipos de floresta perenifólia. Para este estudo foram recolhidas amostras a diferentes profundidades em perfil de solo de pinhais e eucaliptais em diferentes substratos. Cada amostra de solo foi sujeita a diferentes tempos de combustão e foi avaliada a existência, ou não, de diferenças significativas. Os resultados obtidos não apresentam diferenças significativas considerando os diferentes tempos de combustão em cada amostra, apesar de existirem diferenças muito significativas em termos de matéria orgânica nas diferentes amostras.

Palavras-chave: Matéria Orgânica, solos, pinhais, eucaliptais.

\section{ABSTRACT}

Climate change related to global warming called attention to the urgency on identifying and implement adaptation and mitigation strategies, aiming to reduce potential impacts. In this context, measures dedicated to reduce greenhouse gases emissions assumed great importance. In the case of carbon dioxide, beyond emissions reduction, several measures have been also focused on increasing sink capacity, namely in the soil. In this context, different studies evaluated the amount of organic carbon in different soil conditions and land use, having by reference different methodologies. In the case of those that based the assessment on the combustion of organic matter, different parameters in terms of time length and temperatures have been used, limiting the possibility of comparing results. This work aims to assess if time length has clear interference on results, using by reference different soil types and forests. For this study soil samples were collected at different depths on different geological substrates and for the two most common evergreen forests extant on Centro Region of Portugal. Each sample was used to obtain organic carbon values, based on the combustion approach, and having by reference three different time lengths $(3,4,6$ hours). The analysis of results shows that no significant differences were found on results considering time length used for combustion, despite significant differences in terms of organic matter among soil types.

Key-words: Organic Matter, soils, pine forest, eucalyptus forest.

\section{Introdução}

O aumento da temperatura atmosférica média global tem sido associada ao aumento da concentração de gases com efeito de estufa, nomeadamente o dióxido de carbono (Ábrego, 2018; Digiantonio et al., 2019).

A emissão antropogénica de dióxido de carbono é apontada como a principal responsável por este fenómeno físico, denominado aquecimento global (Florides Christodoulides, 2009). Dos muitos impactos diretos e indiretos do aquecimento global consta a subida média do 
nível do mar, o aumento da frequência e intensidade de eventos climáticos extremos, ou a perturbação grave de ecossistemas que, aliado à degradação e fragmentação promovida pelas atividades humanas, poderá contribuir de forma ativa para a extinção de espécies (Florides Christodoulides, 2009; Grimm et al., 2013; Heimann Reichstein, 2008).

Dados da National Oceanic and Atmospheric Administration (2019) indicam que nos últimos 800000 anos a quantidade de dióxido de carbono na atmosfera nunca ultrapassou as 300 partes por milhão (ppm). Situação esta que se altera no período pós-revolução industrial, em que o aumento da libertação do dióxido de carbono para a atmosfera contribui para superar este limiar, com valores que na atualidade superam os 410 ppm e com tendência para aumentar (412,55 ppm - valor registado em Agosto de 2020).

O maior reservatório de carbono do planeta são os oceanos, seguidos dos solos, que retêm 2 a 3 vezes mais carbono do que a atmosfera e (Clara et al., 2017; Keenan Williams, 2018; Lefèvre, 2005; Li et al., 2019; Yang et al., 2019). Os solos constituem, assim, o maior reservatório de carbono terrestre (Petrokofsky et al., 2012; Köchy et al., 2015), sendo também o mais estável (Vagen \& Winowiecki, 2013; (Yang et al., 2019; Zhang et al., 2019).

No âmbito do presente estudo é crucial a distinção entre termos semelhantes, mas com significados muito diferentes, tais como: matéria orgânica, carbono orgânico e carbono inorgânico dos solos. Embora o carbono orgânico seja um componente derivado da matéria orgânica, o carbono orgânico possui elementos que o diferenciam da matéria orgânica. A matéria orgânica tem a sua origem principalmente nas plantas, encontra-se sob a superfície do solo contendo raizes, fauna e microorganismos vivos (Hoyle, 2013), em vários estados de decomposição com tamanhos inferiores a $2 \mathrm{~mm}$ (Clara et al., 2017). O carbono orgânico do solo constitui cerca de 55 a 60\% da massa da matéria orgânica (Clara et al., 2017; Hoyle, 2013). A mesma matéria orgânica é composta por carbono e outros elementos químicos como hidrogênio, oxigênio e pequenas quantidades de nitrogênio, fósforo, enxofre, potássio, cálcio e magnésio (Hoyle, 2013). Em regra, o carbono orgânico do solo é calculado através da análise de amostras recolhidas a profundidades compreendidas entre os $0-30 \mathrm{~cm}$, profundidade recomendada pelo Intergovernmental Panel on Climate Change (IPCC), pois é nas camadas superiores do solo que a atividade das raízes mais se concentra (Ravindranath and Ostwald, 2008). As formas inorgânicas de carbono são derivadas essencialmente de fontes geológicas do solo. As formas inorgânicas de carbono estão presentes em solos e 
sedimentos ricos em carbonatos. Os dois cabonatos minerais mais comuns são os cabonatos de cálcio $\left(\mathrm{CaCO}_{3}\right)$ e dolomite $\left[\mathrm{CaMg}\left(\mathrm{CO}_{3}\right)_{2}\right]$, embora outras formas possam estar presentes (por exemplo, siderite, $\mathrm{FeCO}_{3}$ ) (Schumacher, 2002).

Nas últimas décadas, a avaliação do carbono orgânico do solo ganhou importância no âmbito da definição de medidas dedicadas à mitigação dos efeitos das alterações climáticas, assumindo-se como um parâmetro importante a considerar na lista de opções classificadas como "Nature Based-Solutions" (Périé Ouimet, 2007), nomeadamente através do aumento de capacidade de sequestro de carbono. Este parâmetro assume grande significado, uma vez que as ações que promovem o aumento da capacidade de sequestro, como é o caso da reflorestação, contribuem de diversas formas para mitigar os efeitos das alterações climáticas, nomeadamente pela redução da erosão dos solos, aumento do potencial edáfico, ou até pelo reforço da infiltração e recarga dos aquíferos, contribuindo para um aumento da disponibilidade hídrica (Clara et al., 2017; Demessie et al., 2017).

A nível nacional, há o compromisso em garantir a neutralidade carbónica até 2050, em linha com os objetivos europeus (ENAAC2020, 2010; PNPOT, 2019). Para se alcançar este objetivo, a prioridade passa por diminuir a emissão e reforçar a capacidade de sequestro de carbono. Esse aumento do sequestro está baseado essencialmente na implementação de medidas baseadas na aplicação de soluções naturais "Nature Based-Solutions" com o objetivo de mitigar os efeitos de uma mudança climática global ou regional (Cotrufo et al., 1998).

São diversas as propostas metodológicas para o cálculo do carbono orgânico no solo, nomeadamente por espectroscopia através de infravermelho (Cohen et al., 2005), através da combustão (Konare et al., 2010) ou por reação química utilizando peróxido de hidrogénio (Schumacher, 2002). As propostas metodológicas baseadas na combustão são muito variáveis em relação ao tempo e temperatura a utilizar no processo, sendo possível encontrar propostas entre os $375^{\circ} \mathrm{C}$ e os $800^{\circ} \mathrm{C}$ nos mais variados tempos de combustão (Bisutti et al., 2004). Ainda que se identifique a prevalência na utilização de valores de temperatura entre os $500^{\circ} \mathrm{C}$ e os $600^{\circ} \mathrm{C}$ (e.g.: Jessen et al., 2018), existem autores que defendem que são necessárias temperaturas mais elevadas. Também o tempo de combustão utilizado difere de forma significativa, podendo variar entre as 4 horas (Jessen et al., 2018) e as 16 horas (Konare et al.,2010). 
Uma vez que diversos trabalhos diferem nos parâmetros de referência para a combustão (tempo e temperatura de combustão), este trabalho procura avaliar se o tempo de combustão é um fator determinante para os valores de carbono orgânico obtidos, utilizando como referência diferentes tipos de solo e de floresta. Uma vez que muitos dos trabalhos apresentam resultados com baixo teor de matéria orgânica, neste trabalho optou-se por amostrar solos com valores de matéria orgânica distintos, permitindo avaliar se esta condição é importante na hora de se definir o tempo de combustão de referência.

\section{Metodologia}

No presente estudo, a determinação do carbono orgânico no solo foi elaborada através do método da combustão em mufla (Forno cerâmico Modelo E2 - sonda tipo K 1300 C Barracha, Lda), por ser considerado um método simples, rápido, fiável e utilizado por diversos autores (Jensen et al., 2018).

Optou-se por uma temperatura de combustão constante de 500 으, uma vez que a temperaturas superiores pode observar-se a destruição de matéria não orgânica (desidroxilação das argilas), o que resultaria em maiores perdas de peso, resultando no enviesamento dos resultados (Bisutti et al., 2004; Konare et al., 2010), e a utilização de temperaturas inferiores poderia não assegurar a combustão total de toda a matéria orgânica presente nas amostras (Bisutti et al., 2004). Considerando a diversidade de propostas em termos de tempos de combustão (Abella Zimmer, 2007; Bisutti et al., 2004; De Vos et al., 2005), neste estudo definiram-se 3 tempos de referência: 3, 4 e 6 horas.

Como os resultados de diferentes estudos apresentam baixos valores de carbono orgânico, indicando baixos valores de matéria orgânica, neste estudo optamos por realizar amostragem em tipos de solos para os quais se espera grandes diferenças em termos de matéria orgânica, no sentido de confirmar a necessidade de se considerar diferentes condições (tempo e temperatura) dependendo dos valores de matéria orgânica do solo. Em termos de amostragem, foram recolhidas amostras a diferentes profundidades $(10,20$ e 30 $\mathrm{cm}$ ), em diferentes substratos (complexo xisto-grauváquico e sistema dunar) e diferentes tipos de floresta adulta perenifólia (pinhal bravo e eucaliptal). A opção por estes dois tipos de floresta perenifólia está relacionada com a sua representatividade em Portugal. A opção 
por recolher amostras no complexo xisto-grauváquico e em sistemas dunares está determinada pela identificação de condições edáficas muito diferenciadas, nomeadamente em termos de concentração de matéria orgânica.

A amostragem foi realizada no setor ocidental da Região Centro, uma área caraterizada por grande diversidade litológica e tipos de florestas, onde domina um clima de padrão mediterrâneo, com verões quentes e secos. Os valores de precipitação total média anual nesta área variam entre os $800 \mathrm{~mm}$ e os $1200 \mathrm{~mm}$, e valores de défice hídrico moderado a elevado (Figueiredo et al., 2018). No total foram recolhidas 72 amostras em florestas adultas perenifólias associadas a reflorestação: 18 amostras recolhidas em eucaliptais adultos instalados em cambissolos húmicos em substrato xistento do complexo xistograuváquico (Serra da Aveleira: perfis A1 e A2); 27 amostras em pinhais adultos instalados em cambissolos húmicos em substrato xistento do complexo xisto-grauváquico (Serra da Lousã: perfis L1, L2 e L3); 27 amostras em Pinhal adulto instalado em arenossolos de sistema dunar (Mata Nacional de Leiria: perfis PL1, PL2 e PL3).

As amostras de solo foram recolhidas longitudinalmente a diferentes profundidades $(10,20$, $30 \mathrm{~cm}$ ) recorrendo a cincho (12 cm de comprimento e $6 \mathrm{~cm}$ de diâmetro), após preparação de perfil de solo em cada local de amostragem, seguindo as indicações da amostragem padrão (IPCC, 2006).

Em termos de preparação, as amostras foram secas numa estufa pelo período de 24 horas a uma temperatura constante de 105 C (Abella Zimmer, 2007; Jensen et al., 2018; Périé Ouimet, 2007; J.-P. Wang et al., 2013). Foram, também, eliminados os macrorrestos vegetais e separados os materiais mais grosseiros utilizando um crivo de $2 \mathrm{~mm}$, enquanto os agregados de solos foram destruídos por ação mecânica. Em cada amostra foi testada a presença de carbonatos com recurso à solução de ácido Clorídrico a 10\% (Abella Zimmer, 2007; Konare et al., 2010), não se tendo observado reação relevante .

Para cada amostra, e utilizando uma balança de precisão (0,001 gr), foram separados 5 gramas de solo em três copos de vidro ( 5 gr em cada), previamente colocados em estufa e arrefecidos em dissecador com sílica (Abella Zimmer, 2007; Jensen et al., 2018; J.-P. Wang et al., 2013). Posteriormente, cada copo de vidro foi sujeito a um tempo de combustão diferente $(3,4$ ou $6 \mathrm{~h})$. 
Decorrido o tempo definido para combustão, as amostras foram colocadas no dissecador, até a temperatura descer para a temperatura ambiente, e seguidamente foram pesadas.

Após a pesagem, foi aplicada a fórmula matemática MO = PASF*100/PASI em que MO (Matéria Orgânica, em percentagem), PASF (Peso da Amostra de solo Final, em gramas) e PASI (Peso da Amostra de Solo Inicial, em gramas). A conversão de matéria orgânica para o cálculo do carbono orgânico seguiu a regra de conversão conhecida, através do fator de multiplicação de 0,58 (Jensen et al., 2018; Périé Ouimet, 2007; Schumacher, 2002).

Para análise de diferenças entre amostras, além do cálculo de medidas estatísticas descritivas (média, desvio padrão, coeficiente de variação), foram também aplicados testes Anova, com o objetivo de avaliar se as diferenças observadas são estatisticamente significativas, depois de confirmada a existência de homogeneidade de variâncias $(p>0,05)$ e normalidade na distribuição $(p>0,05)$ através da aplicação dos testes de Levene e ShapiroWilk respetivamente.

Como o objetivo principal do presente trabalho é responder à questão se o tempo de combustão influencia os resultados, e não propriamente o valor exato de carbono orgânico, a obtenção dos valores de carbono orgânico não teve por referência dados de granulometria, nomeadamente a percentagem de materiais finos, como proposto por alguns autores (De Vos et al., 2005; Jensen et al., 2018; Konare et al., 2010; Rumpel KögelKnabner, 2011; Salehi et al., 2011; X. Wang et al., 2012).

\section{Resultados e Discussão}

Em termos de valores de carbono orgânico, os resultados obtidos indicam que há diferenças significativas entre as amostras recolhidas, o que permite avaliar se há variação nos resultados em função do tempo de combustão considerando amostras com diferentes valores de matéria orgânica.

Os resultados demonstram claramente que as amostras recolhidas no complexo xistograuváquico (A1, A2, L1, L2, L3) apresentam valores substancialmente mais elevados do que as amostras recolhidas em arenossolos do sistema dunar (PL1, PL2 PL3), o que demonstra que o tipo de solo assume maior influência nos valores de carbono orgânico que o tipo de floresta. Na verdade, os valores mais elevados de carbono orgânico e os valores mais baixos 
foram obtidos em perfis associados a parcelas ocupadas por pinhal bravo, estando a diferença apenas no tipo de solo/substrato, cambissolos do complexo xisto-grauváquico e arenossolos de sistemas dunares respetivamente (Figura 1). Enquanto que em cambissolos do complexo xisto-grauváquico em pinhal bravo (Figura 3) os valores médios de carbono orgânico rondam os 10,5\% nos $30 \mathrm{~cm}$ superficiais, já em arenossolos os valores médios rondam os $0.74 \%$ de carbono orgânico. Algumas amostras dos perfis da Serra da Lousã (L1 a L3) aos $10 \mathrm{~cm}$ de profundidade continham valores de carbono orgânico próximos dos $18 \%$. Mesmo à profundidade de $30 \mathrm{~cm}$, algumas amostras registaram valores de carbono orgânico superiores a $14 \%$.

Considerando o comportamento dos valores de carbono orgânico no perfil, e tal como esperado para solos com ocupação florestal, é nos primeiros $10 \mathrm{~cm}$ de profundidade onde se registam os valores mais elevados, verificando-se uma diminuição dos valores com a profundidade (Figura 1).

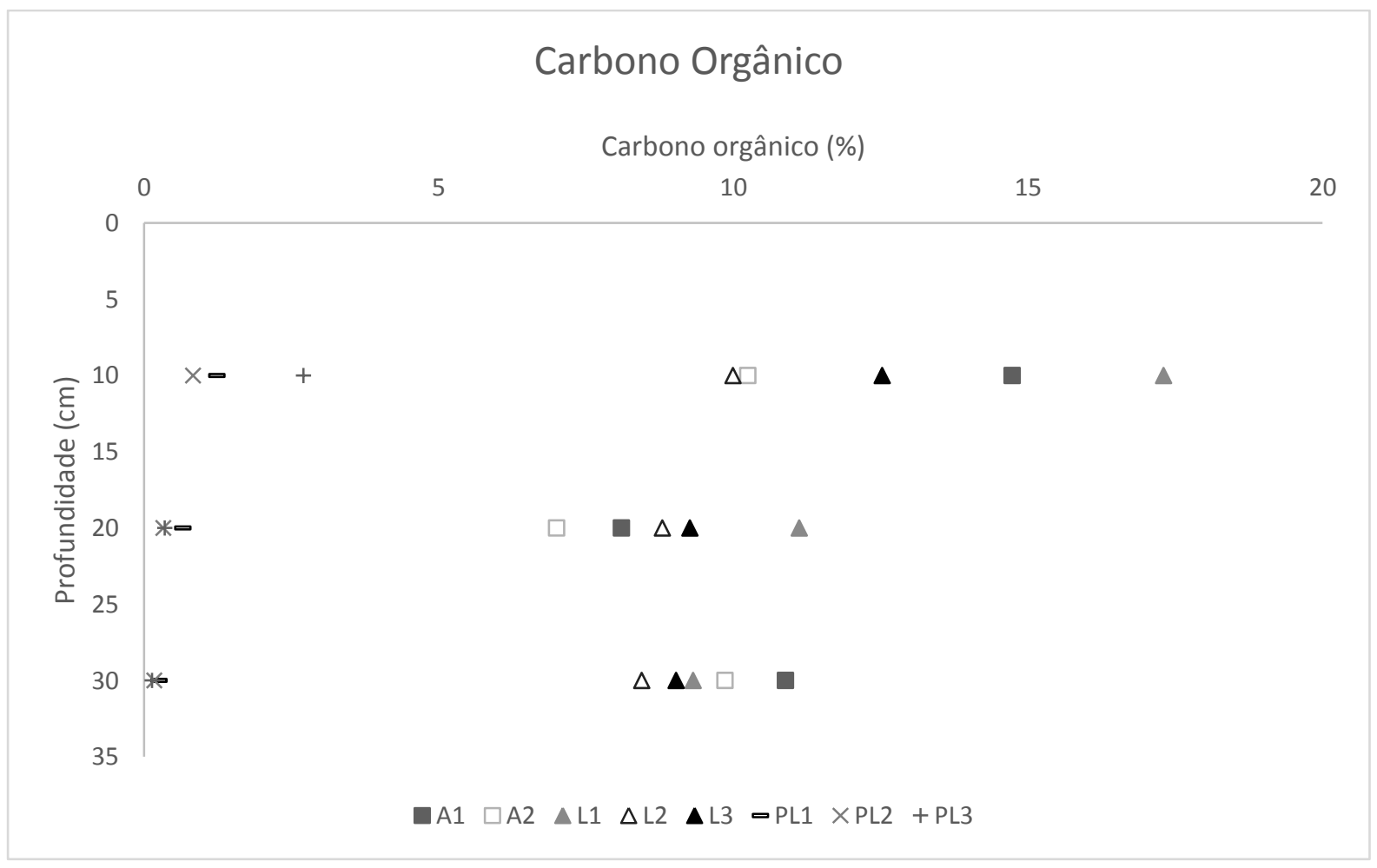

Figura 1: Percentagem de carbono orgânico nos diferentes perfis dos locais de amostragem (Serra da Aveleira A1, A2; Serra da Lousã L1, L2, L3; Pinhal de Leiria PL1, PL2, PL3) .

Aos 20 e 30 centímetros de profundidade os valores de carbono orgânico são mais baixos, com valores próximos entre si ou a decrescer com o aumento de profundidade (Figuras 3 e 
4). A exceção a este padrão está nos locais amostrados na Serra da Aveleira (Figura 2), o que pode estar associado a processos de revolvimento do solo para instalação da floresta. Seria de prever que os valores de carbono orgânico fossem diminuindo com o aumento da profundidade (Rumpel Kögel-Knabner, 2011). Todavia, os dados obtidos não duplicam totalmente este padrão expectável. Este comportamento pode relacionar-se com o processo de instalação da floresta de eucaliptos através da abertura de valas com meios mecânicos, com a subsequente inversão dos horizontes de solo. O recurso a esta técnica na plantação de eucaliptos obriga a mobilizações profundas, que podem atingir os $50 \mathrm{~cm}$ de profundidade, e com elas verificar-se o soterramento dos sobrantes, os quais contribuem para incrementar a matéria orgânica em profundidade.

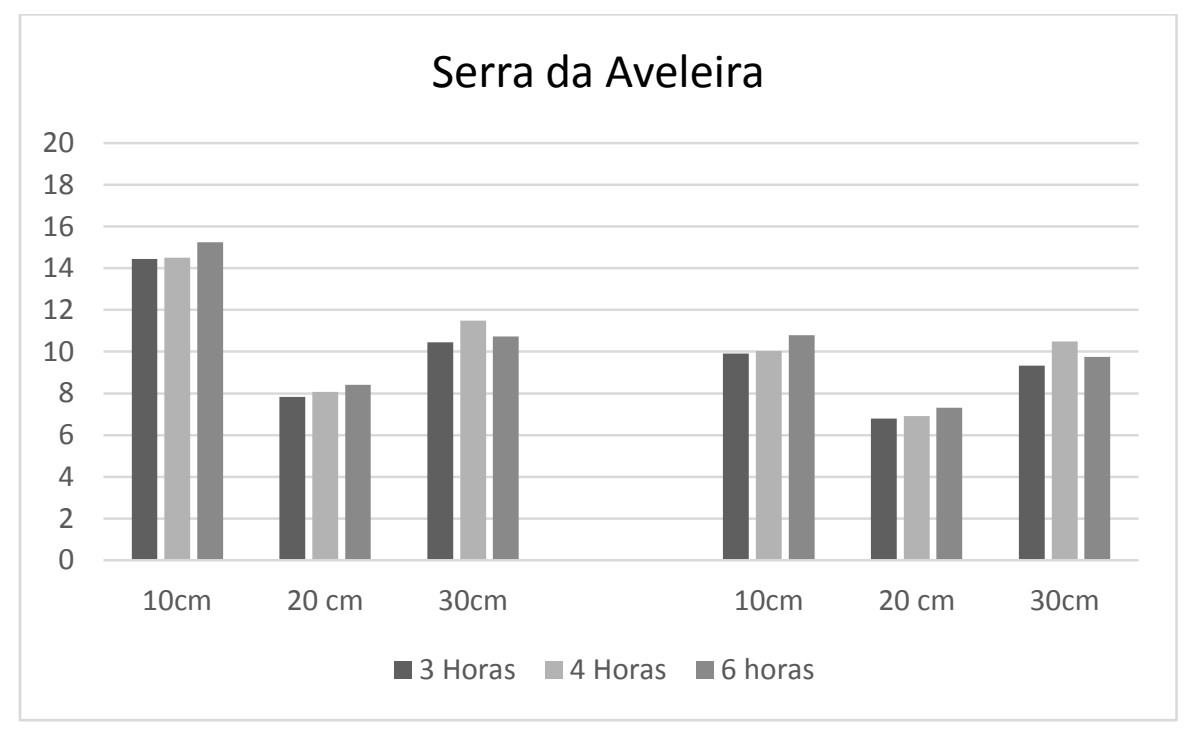

Figura 2: Percentagem de carbono orgânico após combustão a $500^{\circ} \mathrm{C}$

Contrariamente aos solos da Serra da Aveleira (A1, A2) (Fig. 2) e da Serra da Lousã (L1, L2, L3) (Fig. 3), os solos da Mata Nacional de Leiria (PL1, PL2, PL3) (Fig.4) são pouco orgânicos, apresentando uma percentagem muito baixa de carbono orgânico (Figura 4), um padrão comum em solos instalados em dunas secundárias (Sevink, 1991), como é o caso.

Apesar das diferenças identificadas em termos de quantidade de carbono orgânico nas amostras utilizadas (Figuras 2, 3 e 4), o que remete para diferenças significativas nos valores de matéria orgânica, os resultados do teste Anova permitem constatar que o tempo de combustão não interfere nos valores obtidos, uma vez que não se identificam diferenças significativas ao nível dos resultados considerando diferentes tempos de combustão para cada amostra. Tanto para os perfis com valores mais elevados de matéria orgânica, associados a pinhais adultos instalados em cambissolos de substrato xistento do complexo 
xisto-grauváquico (Serra da Lousã: L1 a L3), como para os perfis de solos com valores mais baixos de carbono orgânico, associados a pinhais adultos instalados em arenossolos de sistemas dunares, não há diferenças importantes nos valores de carbono orgânico em função do tempo de combustão ( $p>0.05)$.

Em termos de perfil, os resultados dos testes ANOVA confirmam a existência de diferenças estatisticamente significativas em termos de carbono orgânico para as 3 profundidades analisadas em função dos pontos de recolha $(p<0.05)$. Os resultados da aplicação do teste Tukey B mostram que nos $20 \mathrm{~cm}$ superficiais os teores de carbono orgânico são semelhantes para os pinhais bravos da Lousã e os eucaliptais da Aveleira, enquanto aos $30 \mathrm{~cm}$ se assinalam diferenças significativas entre as 3 áreas de amostragem. Na verdade, os perfis do Pinhal de Leiria apresentam valores significativamente inferiores, sendo as diferenças mais significativas aos 20 e $30 \mathrm{~cm}$ de profundidade $(p<0.001)$, onde os valores de carbono orgânico são consideravelmente inferiores aos da Serra da Aveleira e Serra da Lousã. Na verdade, os pinhais instalados em arenosolos, além de apresentarem os valores mais baixos de carbono orgânico, apresentam ainda coeficientes de variação mais elevados, indicando maior variação no perfil, o que está associado a um decréscimo muito significativo em profundidade, associado a uma redução significativa da quantidade de matéria orgânica no perfil do solo (Tabelas 1 e 2).

Tabela 1: Média, desvio padrão e coeficiente de variação de carbono orgânico obtidos para os perfis analisados

\begin{tabular}{|l|c|c|c|c|c|c|c|c|}
\hline Tipo de floresta & \multicolumn{3}{|c|}{ Eucaliptais } & \multicolumn{5}{c|}{ Pinhais bravos } \\
\hline Tipo de solo & \multicolumn{3}{|c|}{ cambissolos } & \multicolumn{3}{c|}{ arenossolos } \\
\hline Tipo de substrato & \multicolumn{3}{|c|}{ xistos do complexo xisto-grauváquico } \\
\hline Ponto de Amostra & Aveleira 1 & Aveleira 2 & Lousã 1 & Lousã 2 & Lousã 3 & Leiria 1 & Leiria 2 & Leiria 3 \\
\hline Média Co (\%) & 11,24 & 9,04 & 12,58 & 9,08 & 10,27 & 0,72 & 0,44 & 1,06 \\
\hline Desvio Padrão (\%) & 2,91 & 1,59 & 3,67 & 1,06 & 1,71 & 0,45 & 0,35 & 1,24 \\
\hline Coef. Variação (\%) & 0,15 & 0,10 & 0,17 & 0,07 & 0,10 & 0,37 & 0,33 & 0,68 \\
\hline
\end{tabular}


Tabela 2: Estatísticas descritivas para valores de carbono orgânico nas áreas amostradas

\begin{tabular}{|l|c|c|c|}
\hline \multicolumn{1}{|c|}{ Local de amostragem } & Aveleira & Lousã & Pinhal de Leiria \\
\hline Média (\%) & $\mathbf{1 0} \mathbf{~ c m ~}$ & $\mathbf{1 0} \mathbf{~ c m}$ & $\mathbf{1 0} \mathbf{~ c m}$ \\
\hline Desvio Padrão (\%) & 12,49 & 13,28 & 1,60 \\
\hline Coef. Variação (\%) & 2,49 & 3,24 & 0,87 \\
\hline Profundidade & $\mathbf{2 0} \mathbf{~ c m}$ & $\mathbf{2 0} \mathbf{~ c m}$ & $\mathbf{2 0} \mathbf{~ c m}$ \\
\hline Média (\%) & $\mathbf{7 , 5 5}$ & 9,38 & 0,44 \\
\hline Desvio Padrão (\%) & 0,65 & 1,49 & 0,21 \\
\hline Coef. Variação (\%) & 0,09 & 0,16 & 0,47 \\
\hline Profundidade & $\mathbf{3 0} \mathbf{~ c m}$ & $\mathbf{3 0} \mathbf{~ c m}$ & $\mathbf{3 0} \mathbf{~ c m}$ \\
\hline Média (\%) & 10,37 & 8,93 & 0,19 \\
\hline Desvio Padrão (\%) & 0,75 & 0,64 & 0,10 \\
\hline Coef. Variação(\%) & 0,07 & 0,07 & 0,55 \\
\hline
\end{tabular}

Perante os dados apresentados, conclui-se que não existem diferenças significativas em termos de valores de carbono orgânico obtidos considerando diferentes tempos de combustão (3, 4 e 6 horas) a uma temperatura constante de $500^{\circ} \mathrm{C}$. Estes resultados permitem reduzir o tempo dedicado ao processo de obtenção de dados em laboratório, o que pode significar uma redução significativa quando é necessário processar muitas amostras.

\section{Conclusão}

O carbono orgânico do solo, na estreita dependência da matéria orgânica presente no solo, desempenha importantes funções, uma vez que está relacionado com o fornecimento de bens e serviços vários, quer de aprovisionamento, quer de regulação ou de suporte. 0 significativo aumento da concentração de $\mathrm{CO}_{2}$ na atmosfera, e a sua estreita relação com o aquecimento global, desencadeou um crescente interesse na promoção de medidas com vista ao incremento do seu sequestro no âmbito da definição de medidas de mitigação dos impactes das alterações climáticas. A adequada gestão dos stocks de carbono tem, por isso, constituído motivo de preocupação no contexto das agendas políticas internacionais sobre a 
mitigação das emissões de gases com efeito de estufa. Neste âmbito, diferentes estudos têm dedicado atenção à avaliação da capacidade de sequestro de carbono, propondo diferentes abordagens metodológicas.

Com este trabalho contribuiu-se para a clarificação de um dos aspetos metodológicos associados à avaliação do carbono orgânico presente no solo, tendo em conta a falta de uniformidade em termos de tempos de combustão utilizados na obtenção de resultados pelo método da combustão. Recorrendo a solos com diferentes concentrações de matéria orgânica, foi possível concluir que o tempo de combustão, comparando os resultados de 3 opções também utilizadas noutros estudos (3, 4 e 6 horas), não tem influência significativa na determinação da quantidade de carbono orgânico presente no solo. Esta conclusão permite reduzir o tempo dedicado ao processo de combustão, permitindo aumentar a capacidade de análise, o que pode ser significativo em estudos com elevado número de amostras.

\section{Agradecimentos}

Os autores deste estudo agradecem ao Centro de Estudos em Geografia e Ordenamento do Território da Universidade de Coimbra o apoio atribuído para o processo de recolha de dados em campo.

\section{Referências}

Abella, S. R., \& Zimmer, B. W. (2007). Estimating Organic Carbon from Loss-On-Ignition in Northern Arizona Forest Soils. Soil Science Society of America Journal, 71(2), 545-550. doi:10.2136/sssaj2006.0136

Ábrego, J. F. (2018). The Global Warming and the Possible Solution. Journal of Geoscience and Environment Protection, 6, 118-120. doi:https://doi.org/10.4236/gep.2018.65010

Bisutti, I., Hilke, I., \& Raessler, M. (2004). Determination of total organic carbon - an overview of current methods. TrAC Trends in Analytical Chemistry, 23(10), 716-726. doi:https://doi.org/10.1016/i.trac.2004.09.003

Clara, L., Fatma, R., Viridiana, A., \& Liesl, W. (2017). Soil Organic Carbon: The Hidden Potential. Rome, Italy: Food and Agriculture Organization of the United Nations.

Cohen, M. J., Prenger, J. P., \& DeBusk, W. F. (2005). Visible-Near Infrared Reflectance Spectroscopy for Rapid, Nondestructive Assessment of Wetland Soil Quality. Journal of Environmental Quality, 34(4), 14221434. doi:10.2134/jeq2004.0353 
Cotrufo, M. F., Berg, B., \& Kratz, W. (1998). Increased atmospheric CO2 and litter quality. Environmental Reviews, 6(1), 1-12. doi:10.1139/a97-013

De Vos, B., Vandecasteele, B., Deckers, J., \& Muys, B. (2005). Capability of Loss-on-Ignition as a Predictor of Total Organic Carbon in Non-Calcareous Forest Soils. Communications in Soil Science and Plant Analysis, 36(19-20), 2899-2921. doi:10.1080/00103620500306080

Demessie, A., Singh, B. R., \& Lal, R. (2017). Soil Carbon Sequestration: Ethiopia. Selenium - Soil Enzymes. doi:10.1081/E-ESS3-120053587

Digiantonio, G., Kelley, E., Bayler, E., Christerson, N., Davis, S., Dreflak, K., . . Roohr, P. (2019). 2019 National Oceanic and Atmospheric Administration Science Report. Retrieved from

Florides, G. A., \& Christodoulides, P. (2009). Global warming and carbon dioxide through sciences. Environment International, 35(2), 390-401. doi:https://doi.org/10.1016/i.envint.2008.07.007

Grimm, N. B., Chapin III, F. S., Bierwagen, B., Gonzalez, P., Groffman, P. M., Luo, Y., . . Williamson, C. E. (2013). The impacts of climate change on ecosystem structure and function. Frontiers in Ecology and the Environment, 11(9), 474-482. doi:10.1890/120282

Heimann, M., \& Reichstein, M. (2008). Terrestrial ecosystem carbon dynamics and climate feedbacks. Nature, 451, 289. doi:10.1038/nature06591

Hoyle, F. (2013). Managing Soil Organic Matter: A Pratical Guide (J. Paterson Ed.). Department of Agriculture and Food Western Australia

Jensen, J. L., Christensen, B. T., Schjønning, P., Watts, C. W., \& Munkholm, L. J. (2018). Converting loss-onignition to organic carbon content in arable topsoil: pitfalls and proposed procedure. European Journal of Soil Science, 69(4), 604-612. doi:10.1111/ejss.12558

Keenan, T. F., \& Williams, C. A. (2018). The Terrestrial Carbon Sink. Annual Review of Environment and Resources, 43(1), 219-243. doi:10.1146/annurev-environ-102017-030204

Konare, H., Yost, R. S., Doumbia, M., McCarty, G. W., Jarju, A., \& Kablan, R. (2010). Loss on ignition: Measuring soil organic carbon in soils of the Sahel, West Africa. African Journal of Agricultural Research, 2010 v.5 no.22(no. 22), pp. 3088-3080. doi:10.5897/ajar

Lefèvre, N. (2005). How is the ocean responding to the increase in atmospheric $\mathrm{CO}<\mathrm{sub}>2</$ sub $>$ ? Ocean challenge, 13(3), 17-22.

Li, H., Ilyina, T., Müller, W. A., \& Landschützer, P. (2019). Predicting the variable ocean carbon sink. Science Advances, 5(4), eaav6471. doi:10.1126/sciadv.aav6471

Périé, C., \& Ouimet, R. (2007). Organic carbon, organic matter and bulk density relationships in boreal forest soils. Canadian Journal of Soil Science.

Rumpel, C., \& Kögel-Knabner, I. (2011). Deep soil organic matter-a key but poorly understood component of terrestrial C cycle. Plant and Soil, 338(1), 143-158. doi:10.1007/s11104-010-0391-5

Salehi, M. H., Beni, O. H., Harchegani, H. B., Borujeni, I. E., \& Motaghian, H. R. (2011). Refining Soil Organic Matter Determination by Loss-on-Ignition. Pedosphere, 21(4), 473-482. doi:https://doi.org/10.1016/S1002-0160(11)60149-5

Schumacher, B. A. (2002). Methods for the determination of total organic carbon (toc) in soils and sediments. United States Environmental Protection Agency, Environmental Sciences Division National.

Sevink, J. (1991). Soil development in the coastal dunes and its relation to climate. Landscape Ecology, 6(1), 4956. doi:10.1007/bf00157744

Wang, J.-P., Wang, X.-J., \& Zhang, J. (2013). Evaluating Loss-on-Ignition Method for Determinations of Soil Organic and Inorganic Carbon in Arid Soils of Northwestern China. Pedosphere, 23(5), 593-599. doi:https://doi.org/10.1016/S1002-0160(13)60052-1

Wang, X., Wang, J., \& Zhang, J. (2012). Comparisons of Three Methods for Organic and Inorganic Carbon in Calcareous Soils of Northwestern China. PLOS ONE, 7(8), e44334. doi:10.1371/journal.pone.0044334 
Yang, Y., Tilman, D., Furey, G., \& Lehman, C. (2019). Soil carbon sequestration accelerated by restoration of grassland biodiversity. Nature Communications, 10(1), 718. doi:10.1038/s41467-019-08636-w

Zhang, L., Sun, Y., Song, T., \& Xu, J. (2019). Harvested Wood Products as a Carbon Sink in China, 1900-2016. International Journal of Environmental Research and Public Health, 16(3), 445. 